Journal of the Optical Society of Korea, Vol. 18, No. 5, pp. 507-516 October 2014

\title{
Spectral Reflectivity Recovery from Tristimulus Values using 3D Extrapolation with 3D Interpolation
}

\author{
Bog G. Kim, John S. Wemer², Michael Siminovitch³, Konstantinos Papamichael, \\ Jeongwon $\mathrm{Han}^{4}$, and Soobeen Park ${ }^{2,3,4, *}$ \\ ${ }^{I}$ Department of Physics, Pusan National University, Busan 609-735, Korea \\ ${ }^{2}$ Department of Ophthalmology and Vision Science, University of California, Davis, CA 95817, USA \\ ${ }^{3}$ Design Department, California Lighting Technology Center, University of California, Davis, CA 95618, USA \\ ${ }^{4}$ Department of Housing and Interior design, Pusan National University, Busan 609-735, Korea
}

※ In our paper, there was a typographic mistake of one of authors' name. The author's name should be "Konstantinos Papamichael" not "Kostantinos Papamichael".

*Corresponding author: sobpark@pusan.ac.kr 